\title{
Pekka Koskela
}

\author{
Gaven Martin ${ }^{1}$ Jani Onninen ${ }^{2}$
}

Published online: 16 June 2021

(C) The Author(s), under exclusive licence to Springer Nature Switzerland AG 2021

The issue of Complex Analysis and its Synergies is dedicated to Pekka Koskela on the occasion of his 60th birthday and represents in part the proceedings of the conference, "Latest in Geometric Analysis: A Celebration of Pekka Koskela's 59th birthday." This conference was held on November 24-30. 2019 in Będlewo, Poland. The conference celebrated Pekka's wide ranging and far-reaching impact on the field: not only through his hugely influential publications, but also through his extensive collaborations and his dedication to mentoring doctoral students. Indeed the majority of the speakers were close collaborators of Pekka, some over very extended periods.

Pekka has supervised an impressive $26 \mathrm{Ph} . \mathrm{D}$. theses in mathematics since 2000. Most of these have been in the area of Geometric function theory and Geometric Analysis. He has shown incredible skill in matching students and problems, and some of his former students are now international leaders in these research areas themselves.

As we all know, "it's hard to make predictions, especially about the future." The conference organizers, however, seemed to predict the COVID-19 pandemic already back in 2018. Or, perhaps there was some other reason to celebrate Pekka's 59th birthday, thus allowing an in-person celebration? Coincidence or not, the timing of the conference reflects well, at least in symbolic level, Pekka's ability to identify the more important future trends in Geometric Analysis along with establishing key results. We summarize some of the highlights of his visionary work below.

Together with Juha Heinonen, Pekka initiated the modern theory of analysis on metric spaces in the groundbreaking 1998 paper "Quasiconformal maps in metric spaces with controlled geometry." This work axiomatized several aspects of Euclidean quasiconformal geometry in the setting of

Gaven Martin

G.J.Martin@massey.ac.nz

1 Massey University, Auckland 0632, New Zealand

2 Syracuse University, 215 Carnegie Building, Syracuse, NY 13244-1150, USA metric measure spaces. In particular, inspired by the fundamental theorem of calculus, Heinonen and Koskela proposed the notion of upper gradient as a substitute for the derivative of a function on a metric measure space. It comes as no surprise that this paper is, by a considerable margin, the highest cited journal article in the 2010 MSC classification category 30 (Complex Analysis) as it initiated the modern studies of analysis on metric spaces.

The theory of Sobolev spaces on a metric measure space has far-reaching applications to several areas in mathematics and it is still developing. The Memoir of the American Math. Soc. paper "Sobolev met Poincaré" by Hajłasz and Koskela has been the number one reference to the theory from day one. It not only develops a theory of Sobolev type function spaces on metric measure spaces but also develops applications to Carnot-Carathéodory spaces, subelliptic equations, analysis on topological manifolds, potential theory on infinite graphs, and analysis on fractals. This paper is the second most cited journal article published in 2000, in all of mathematics.

Pekka has also been one of the leaders in the development of the theory of mappings of finite distortion. The paper "Mappings of finite distortion: monotonicity and continuity" by Iwaniec, Koskela, and Onninen started the systematic studies of mappings of finite distortion in geometric function theory in 2001. The theory of mappings of finite distortion arose from the need to extend the ideas and applications of the classical theory of quasiconformal mappings to the degenerate elliptic setting. There one finds concrete applications in materials science, particularly nonlinear elasticity and critical phase phenomena, and in the calculus of variations.

These three journal publications are just a drop in the bucket. Indeed, Pekka is an extremely productive researcher who has published a number of seminal results in the theory of Geometric Analysis. He is a coauthor of two monographs: "Lectures on mappings of finite distortion" with Hencl and "Sobolev spaces on metric spaces: An approach based on upper gradients" with Heinonen, Shanmugalingam, and Tyson. 
As noted above, Pekka's work is very highly cited. According to MathSciNet, Pekka has 180 publications, cited almost 5000 times (as February 2021) by 1400 different authors! He gave an invited talk at the 2000 European Congress of Mathematics and at the analysis section of the 2010 ICM. He was awarded the Väisälä prize in 2001 and was a member of the editorial board of Acta Mathematica, 2007-2014. He has been a chair of the Department of Mathematics at the University of Jyväskylä or a vice dean there for almost ten years. Currently, he is a member of the University Board.
We hope that you will enjoy browsing and reading this issue, and to Pekka we wish a happy birthday. We wanted to put the issue together to encourage him to continue for, at least, another 60 years of inspiring research and diverse contributions to the field. We are also looking forward to seeing him wearing his trademark Tasmanian Devil T-shirts at the next face-to-face conference, and to seing what he and his students are up to! 